\title{
A System for Visualization and Analysis of Online Pedagogical Interactions
}

\author{
André Rei \\ CRACS \& University of Porto \\ Rua do Campo Alegre, 1021/1055 \\ 4169-007 Porto \\ Portugal \\ up201006281@fc.up.pt
}

\author{
Álvaro Figueira \\ CRACS / INESCTEC \& University of \\ Porto \\ Rua Campo Alegre, 1021/1055 \\ 4169-007 Porto, Portugal \\ arf@dcc.fc.up.pt
}

\author{
Luciana Oliveira \\ CEOS.PP Polytechnic of Porto \\ Rua Jaime Lopes Amorim \\ 4465-004 Matosinhos \\ Portugal \\ Igo@eu.ipp.pt
}

\begin{abstract}
We present a system for a dynamic graphical representation of the interactions captured in educational online environments. The system goes beyond interaction between students and teachers, also addressing resource usage or any other entity for which it is possible to create a relation which binds two entities. By defining these relationships between pairs of entities in an online learning environment (Moodle, in our case) our tool creates a graph, where it is possible to apply techniques of social network analysis. This system brings up new possibilities for e-learning as a tool capable of helping the teacher assorting and illustrating the degree of participation and to find the implicit relations between participants, or participants and resources or events.
\end{abstract}

\section{CCS Concepts}

Applied computing $\rightarrow$ E-learning $\bullet$ Human-centered computing $\rightarrow$ Social network analysis $\bullet$ Human-centered computing $\rightarrow$ Empirical studies in visualization

\section{Keywords}

Learning Management System, Virtual Teaching Environment, Social Network Analysis, Social Graph.

\section{INTRODUCTION}

Web 2.0 technologies (and beyond) have enormous potential to shape the way humans learn and teach. The social nature of these technologies is paving a new landscape in the teaching-learning environments. The already old concept of Electronic Learning is now updated into an eager quest for distance collaboration, transmission of information, and building information and knowledge collaboratively no matter how distant learners and tutors are from each other. According to [6], "e-learning provides learners with a rich virtual network where they can exchange ideas and information and create synergies through interactions with other members of the network".

A Learning Management System is a software created to manage user's learning activities, taking into consideration the features that will make this possible [5]. Open source LMSs help institutions to upgrade their educational infrastructure, allowing teachers to create

\footnotetext{
Permission to make digital or hard copies of all or part of this work for personal or classroom use is granted without fee provided that copies are not made or distributed for profit or commercial advantage and that copies bear this notice and the full citation on the first page. To copy otherwise, or republish, to post on servers or to redistribute to lists, requires prior specific permission and/or a fee.

Conference'10, Month 1-2, 2010, City, State, Country.

Copyright 2010 ACM 1-58113-000-0/00/0010 _..\$15.00.

DOI: http://dx.doi.org/10.1145/12345.67890
}

personalized virtual teaching-learning environments within elearning courses, in a way that the educational goals are met in the best possible way.

Social Network Analysis (SNA) has proven to be extremely powerful at describing and analyzing network behaviors in business, economics and medicine, but its application to e-learning has been relatively limited [6]. SNA comprises the mapping and metrics applied to relationships and flows between individuals. Historically, network nodes constitute persons, while the links represent the relationships between the nodes. As SNA provides a mathematical and visual analysis of human relationships [8], SNA may also be particularly well suited to studying e-learning environments, providing valuable information for analyzing the social aspects of the learning process. Understanding the social dimension of learning as it becomes the focus of many areas of education research, making SNA a tool of central importance in the current learning analytics [7].

Recently, [6] carried out a large study focusing on the use of SNA in e-Learning, in order to understand how SNA can benefit elearning and its impacts, as well as the state of the art and the directions for future studies in this area. From the 37 studies covered in their research, only 14 examined an LMS or Content Management System (CMS). In these cases, generated data allowed analysis of the relationships between teacher and learners. This suggests that, although it is an emerging area and such studies are being published with increasing frequency, they are still in very early stages. As a consequence, we address the detected problem, and a system for visualizing and analyzing online social interactions, integrated in an LMS, is proposed.

The proposed system consists, therefore, of a visualization and analysis module which features a representation of a social graph and a control panel for analysis of the graph with SNA techniques and common metrics. Contrary to many previous proposals [1], [3], and [4], in this work we did not restrict the system to display interactions between course participants (students and teacher), and took it a step further by enhancing it to depict any interaction that occurred, and that could be automatically captured, during an online course. Therefore, resource usage can also be displayed. This approach is in line with recent research, from [9] and [2], for example.

The chosen LMS for the basis of the development of the system was Moodle, because it is one of the most widely used open source LMSs, it has a friendly customizable graphical user interface with good communication tools, and the documentation is easily accessible [5]. The system aims to be flexible and to serve diverse educational situations. In this way, it can be used to represent and analyze various aspects of a virtual teaching-learning environment, 
such as the relationships between teacher and students, activities and events.

\section{SYSTEM'S ARCHITECTURE}

The architecture of our developed system is illustrated in Figure 1. The first diagram on the upper left corner represents the Moodle system. Moodle is offered in a package for installations in Windows or Linux environments, which additionally contains other programs required for the Moodle system execution, such as Apache, MySQL and PHP.

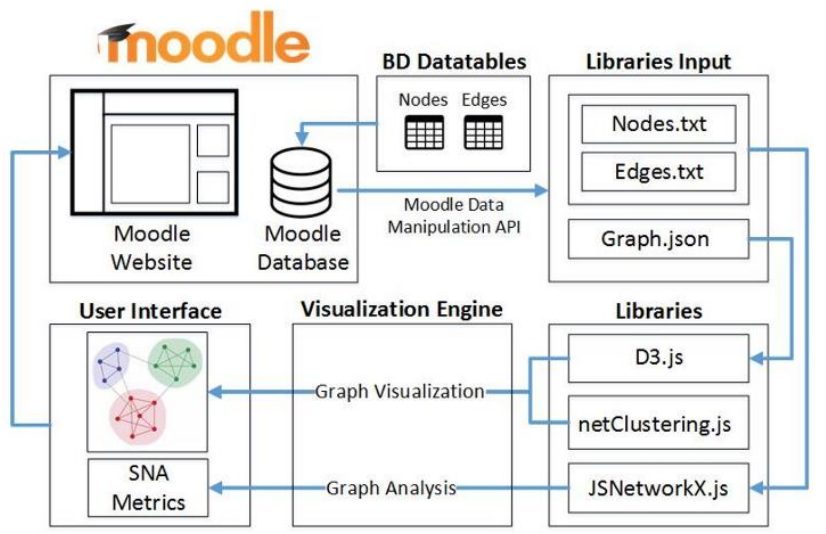

Figure 1. System's architecture.

Two tables were created in the Moodle database, called "moodlesna_nodes" and "moodlesna_links". These tables contain information about the nodes and the edges of the graph, respectively.

Using the Moodle Data Manipulation API, the database connection is made using the get_records_sql() method, which accepts a SQL query as parameter. This method is executed twice: once to get the data from the table of nodes, and another to get the data from the table of edges. The data in these two queries is filtered by the "course_id" column, which represents the id of a course. The two tables share this column, in order to bind each record being associated to a course. When the user selects a course in the Moodle website, a graph is formed with the nodes and edges that have the "course_id" equal to the id of the chosen course.

With the obtained data, three files are then created: "Graph.json", "Nodes.txt" and "Edges.txt". The first one will serve as input for the D3.js library, which creates a force-directed graph from a JSON file. The last two files, serve as input for the JSNetworkX library. In this library, an empty graph is created, and later the nodes and edges are added, with the methods addNodesFrom() and addEdgesFrom(). The first method receives the "Nodes file" as parameter, while the second method receives the "Edges file".

The D3.js library is responsible for creating and displaying the social graph. Since this library does not contain social network analysis features, we used another one which provides this kind of resources: the JSNetworkX library. In this library, during execution, an internal graph is created with the same data as the D3.js library, but without visual representation of it. This graph is required to apply all social network methods and metrics, in which the results are represented in the D3.js library. The netClustering.js library applies a community detection algorithm to the D3.js graph.

\section{USER INTERFACE}

An overview of the user interface is represented in Figure 2. After choosing a course (which should be previously available in Moodle), the user is presented with that control panel. The graph represents the data of the nodes and edges from the chosen course, and is stored in the Moodle database. The graphical interface is split into three main regions as depicted in Figure 2: A) the Social Graph area in the upper left corner; B) the Dashboard in the upper right corner and C) the Graph Properties and metrics, below the other two regions.

In region $\mathbf{A}$, the dashboard allows the user to dynamically interact with the graph, and at the same time, visualizing different characteristics and properties for each node. In this region of the control panel, user interactions are expanded to detect when the user hovers a node, in which case the system presents a grey square in section with metrics about the hovered node. In the provided example, as the orange node was hovered is was possible to see the correspondent Moodle name ("Fantine"), the degree (15), the clustering coefficient $(0.31)$, the eigenvector centrality (0.09) and the betweenness centrality $(0.13)$.

Region $\mathbf{B}$ is devoted to query the system according to common network properties such as the shortest path or network subcommunities. The results of the input parameters are translated into graph representations that overlap the graph in region A. For instance, if we were to find the shortest path between two nodes, then all the nodes in the graph would be blurred apart from these nodes that integrate the path, which would be highlighted. This representation allows a much faster understanding of the dynamic of the net. Accordingly, if we were to detect communities or cliques, only the ones identified by the system would be highlighted.

In region $\mathbf{C}$ we aggregated all the static properties of the graph, that is, the properties related to the all structure of the graph, such as the number of nodes or the graph density. The density measures the level of cohesion of the graph. A dense graph is a graph in which the number of edges is close to the maximum possible number of different connections. In a teaching-learning environment this could allow to measure the cohesion of a class. The number of clusters identify how many sub-groups were formed in the class, and the number of cliques indicate the number of very cohesive sub-groups or communities were each member must have an edge connection to all the others.

\subsection{Mouse Interaction}

The position of the nodes and edges in the graph is not static. The user can drag any node, which in turn drags its connecting edges and, consequently, changes the shape of the graph. The graph can also be moved as a whole, in any direction. Moreover, using the mouse wheel, it is possible to zoom in and out of the visualization of the graph.

When the control panel in Figure 2 loads, the graph node's sizes are different. This is due to the fact that their size is proportional to its degree, therefore the greater the degree of the node, the greater its size. If the user hovers a node with the mouse, a small box appears, which contains information about the selected node, such as its name, degree, Clustering Coefficient, Eigenvector Centrality, and Betweenness Centrality, as showed in Figure 1, section A. 


\section{Moodle Social Graph}

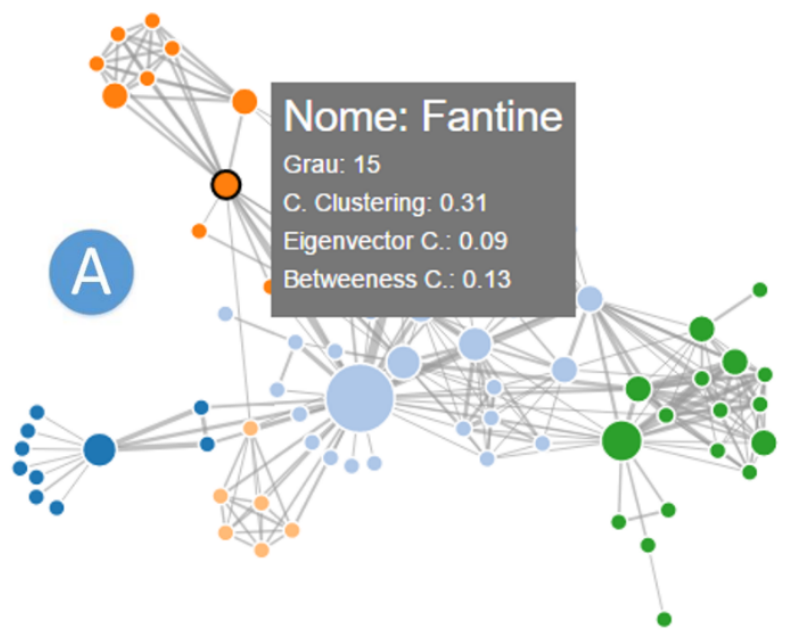

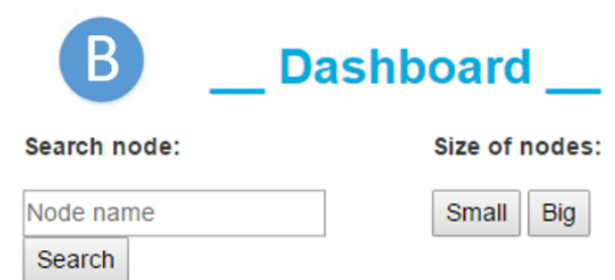

Detection of student communities:

Calculate clusters

Shortest Path between 2 nodes

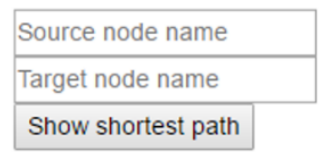

Choothe the number of a cluster between 1 and 5 :

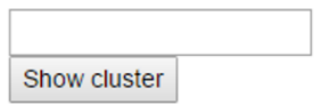

Choose the clique size between 2 and 10

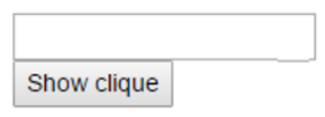

\begin{tabular}{l} 
Graph Properties: \\
$\mathbf{N}^{\circ}$ of nodes: \\
\hline 77 \\
$\mathrm{~N}^{\circ}$ of edges: \\
\hline 254 \\
\hline
\end{tabular}

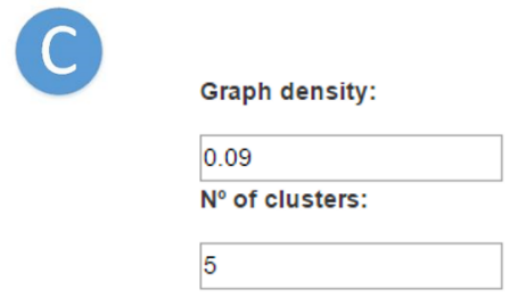

$\mathrm{N}^{\circ}$ of maximum cliques

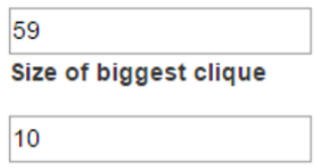

Figure 2. The course control panel.

With a double click on a node, the user can see his direct neighbors and respective edges. After the click, only these nodes and edges become visible, leaving the remaining graph with reduced opacity. With a new double click on the node, the graph returns to normal.

\subsection{Dashboard Interaction}

The dashboard consists of a series of input boxes and buttons grouped by specific actions. The buttons trigger changes in the graph according to the desired action view. In the upper section of the Dashboard, the teacher can choose the name of a node, and see his/her position in the network, as well as change the size of the nodes according to their preferred visualization.

The section below is related to the detection of online student communities. When the screen of Figure 2 appears for the first time, all nodes of the graph have the same color. If the user clicks the "Show Clusters" button, then the "Clauset, Newman and Moore Communities Detection" algorithm [10] is applied to the graph.

This algorithm assigns a property to each node that defines the cluster to which it belongs. This property is an integer used to define the color of the node in the graph drawing. The result is a graph with colored nodes according to the cluster they belong to, such as the graph represented in Figure 2. After the graph is redesigned to represent clusters, the user can choose the number of a particular cluster, and only see that cluster for a period of time, in which all the remaining edges and edges are reduced in opacity. Figure 3 represents the shortest path between two nodes of the graph, for a given input of two node names (in this case: "Count" and "Mabeuf").

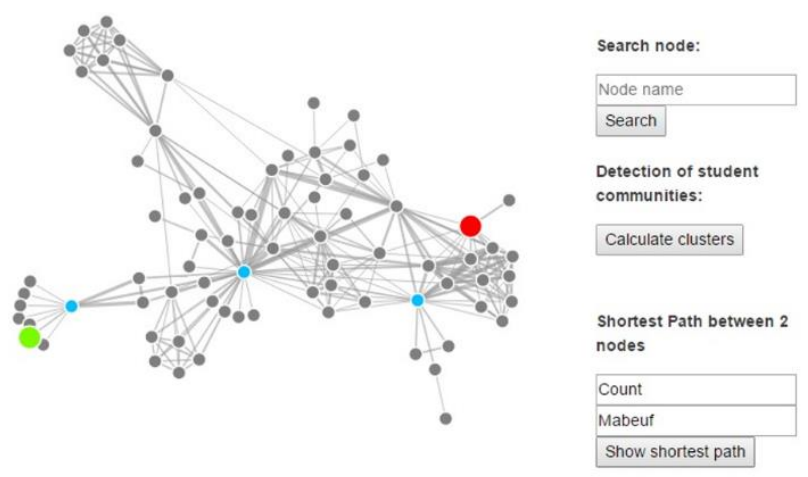

Figure 3. Shortest path between two nodes.

In the first input box, the user chooses the name of the source node and in the second, the name of the target node. By clicking the "Shortest Path" button, the tonality of all nodes changes to gray, except for the source node which is depicted in green, and the target node, depicted in red, and the nodes that comprise the shortest path between the two previous ones, in blue. Using this representation, the teacher can obtain important knowledge about the 'physical' distance between the source node and the target node in the network, as well as the absolute logical location of the nodes in the whole net. In the example given in Figure 3, we can see that only 4 
hops separate the source node from the target node. It is also important to see that these intermediate hops do pass through highdegree nodes and some probable sub-communities.

The dashboard can also be used to check if there are cliques of a certain size in the graph. The number entered in the input box should be between two and the largest clique size of the graph, calculated previously and shown below in the Properties section of the graph (cf. Figure 2, section C). A clique consists of a complete subgraph. In the context of a social network, we can identify a group of people in which everyone talks to each other or a set of educational resources that have been used together (i.e., there might be a stronger dependency from those resources, than from the remaining ones). When the user chooses a value in this range and clicks the "Show Click" button, if there are cliques with that size, the first clique of this size is represented in orange and the remaining nodes are represented in gray. By clicking the button again, the next clique will be shown and so on. Additionally, information on the number of cliques that exists with the given input size is also displayed.

\section{DISCUSSION}

The representation of the shortest path between two nodes can serve several educational purposes. For example, if the interactions are between students, it shows the elements involved in the conversation; on the other hand, if the graph represents resources and duration of their use, then it can display the order in which the resources were "consumed" and how much time the students spent using them.

The main issue here is that social network analysis and the correspondent graphic visualization is not restricted to analyzing interactions between people. Despite this being the traditional case, in many e-learning situations there are latent relations between different entities which, through a graphical representation, emerge as important and insightful (one good example is [11]).

Another important issue to stress it that some other works have been depicting and representing online interaction that occur in Moodle activities, namely in the forum activity ([3][4]) but this is also possible to be extended into social networks which are used in pedagogic contexts, as has been shown in 0 . Nevertheless, the theory behind the development of our system is solid, the graphical representations are generic and therefore, there is a multitude of sources for input data that can be used by our system to be analyzed using the proposed tool.

\section{CONCLUSION}

In this paper, we have presented a system for visualization and analysis of online interactions of people and resources, integrated into the Moodle LMS. This system allows to combine the functionalities of an LMS for educational purposes with SNA techniques, for the study of human relationships in virtual teaching environments. Teachers and students are provided with the best educational diagnosis and with opportunities and weakness in their teaching environments.

As shown in [6], integrating SNA and e-Learning platforms is an area requiring research. The presented system is still in an early stage, so we aim, in a future work to implement important new features. The JSNetworkX library API is at an early stage of its development, so it is expected that over time new algorithms and SNA methods will emerge, which in turn can be integrated into the presented system. It is also possible to implement temporal analysis of the graph, in order to visualize the evolution of the network over time. Finally, we intent to make the system as generic as possible, to be able to receive data from various sources, like social network groups, blogs or forums.

\section{ACKNOWLEDGMENTS}

This work is supported by the ERDF - European Regional Development Fund through the COMPETE Programme (operational programme for competitiveness) and by National Funds through the FCT (Portuguese Foundation for Science and Technology) within project «Reminds/ UTAP-ICDT/EEICTP/0022/2014»

\section{REFERENCES}

[1] Figueira, Á. and Laranjeiro, J., 2008, October. Work in progress - iGraphs for characterization of online communities. In Frontiers in Education Conference, 2008. FIE 2008. 38th Annual (pp. F4E-13). IEEE.

[2] Figueira, A., 2015. Predicting results from interaction patterns during online group work. In Design for Teaching and Learning in a Networked World (pp. 414-419). Springer.

[3] Silva, A. and Figueira, Á., 2012, April. Depicting online interactions in learning communities. In Global Engineering Education Conf. (EDUCON), 2012 (pp. 1-8). IEEE

[4] Silva, A. and Figueira, Á., 2012, July. Visual analysis of online interactions through social network patterns. In Advanced Learning Technologies (ICALT), 2012 IEEE 12th International Conference on (pp. 639-641). IEEE.

[5] Cavus, N. and Zabadi, T., 2014. A comparison of open source learning management systems. Procedia-Social and Behavioral Sciences, 143, pp.521-526.

[6] Cela, K. L., Sicilia, M. Á., \& Sánchez, S. (2015). Social network analysis in e-learning environments: A Preliminary systematic review. Educational Psychology Review, 27(1), 219-246.

[7] Dawson, S., 2010. 'Seeing'the learning community: An exploration of the development of a resource for monitoring online student networking. British Journal of Educational Technology, 41(5), pp.736-752.

[8] Jamali, M. and Abolhassani, H., 2006, December. Different aspects of social network analysis. In Web Intelligence, 2006. WI 2006. IEEE/WIC/ACM International Conference on (pp. 66-72). IEEE.

[9] Ziebarth, S., Chounta, I.A. and Hoppe, H.U., 2015. Resource access patterns in exam preparation activities. In Design for Teaching and Learning in a Networked World (pp. 497-502). Springer International Publishing.

[10] Clauset, A., Newman, M.E. and Moore, C., 2004. Finding community structure in very large networks. Physical review E, 70(6), p.066111.

[11] Figueira, Á., 2017, April. Communication and resource usage analysis in online environments: An integrated social network analysis and data mining perspective. In Global Engineering Education Conference (EDUCON), 2017 IEEE (pp. 1027-1032). IEEE.

Oliveira, L. and Figueira, Á., 2016. EduBridge Social-Bridging Social Networks and Learning Management Systems. In CSEDU (1) (pp. 162-171) 